Growth and spatially resolved luminescence of low dimensional structures in sintered $\mathrm{ZnO}$

This content has been downloaded from IOPscience. Please scroll down to see the full text. 2005 Nanotechnology 16931

(http://iopscience.iop.org/0957-4484/16/6/051)

View the table of contents for this issue, or go to the journal homepage for more

Download details:

IP Address: 147.96.14.16

This content was downloaded on 10/10/2013 at 13:20

Please note that terms and conditions apply. 


\title{
Growth and spatially resolved luminescence of low dimensional structures in sintered $\mathrm{ZnO}$
}

\author{
J Grym, P Fernández ${ }^{1}$ and J Piqueras \\ Departamento de Física de Materiales, Facultad de Ciencias Físicas, Universidad \\ Complutense de Madrid, 28040, Madrid, Spain \\ E-mail: arana@fis.ucm.es
}

Received 26 January 2005, in final form 10 March 2005

Published 19 April 2005

Online at stacks.iop.org/Nano/16/931

\begin{abstract}
Sintering of $\mathrm{ZnO}$ pressed powder under Ar flow at temperatures between 1250 and $1300{ }^{\circ} \mathrm{C}$ leads to the formation of elongated microstructures and nanostructures, with different morphologies, on the sample surface. Rods and needles with cross-sectional dimensions ranging from tens of nanometres to several tens of microns and up to hundreds of microns in length are obtained. In an advanced stage of growth, nanoneedles are frequently arranged in bundles, forming the walls of tubes with different cross-sectional dimensions. In addition, microcombs and microfeathers consisting of well oriented nanoneedles are observed. Cathodoluminescence (CL) in the scanning electron microscope (SEM) has been used to characterize the structures grown. The formation of the elongated structures causes spectral changes, in particular an enhancement of the green-orange luminescence. High CL emission from the internal surface of the tubes has been observed.
\end{abstract}

\section{Introduction}

Fabrication of low dimensional elongated semiconductor structures such as nanowires, nanotubes and nanorods is a subject of increasing interest due to their potential use in optoelectronics, sensor devices and other technological applications. Many low dimensional structures have already been reported in several semiconductor materials and, in particular, an intense activity has been carried out in the development of elongated nanostructures of $\mathrm{ZnO}$ [1-10]. This is partly related to the great interest of this material for photonic applications due to its wide direct bandgap (3.37 eV) and large exciton binding energy $(60 \mathrm{meV})$ enabling optical lasing at room temperature [11-13].

Thermal evaporation of the semiconductor powder and deposition on a substrate under a gas flow is an often reported method for growing low dimensional structures. In some cases the substrate is covered with a film, usually of gold, which acts as a catalyst. Nanowires, rods or tubes of $\mathrm{ZnO}$ have been grown by deposition on different substrates,

1 Author to whom any correspondence should be addressed. without catalyst e.g. [1, 3, 7-10] or with catalyst $[2,4,14]$ as well as by oxidation of $\mathrm{Zn}$ under oxygen flow [5, 15]. In this work, a different method for growing elongated nanostructures and microstructures of $\mathrm{ZnO}$, namely sintering of compacted powder under an argon flow, has been used. This method, which has been recently reported for nanostructures of $\mathrm{CdSe}$ [16] and $\mathrm{SnO}_{2}$ [17], leads to the growth of the structures directly on the sample surface, which acts as source as well as substrate. In this work, the experimental conditions, such as temperature, time of treatment and flow rate, leading to the growth of the elongated structures of $\mathrm{ZnO}$ have been determined and the morphology and luminescence of the obtained nanostructures and microstructures have been investigated by scanning electron microscopy (SEM) and cathodoluminescence (CL) in the SEM respectively.

\section{Experimental details}

The starting material was commercially available $\mathrm{ZnO}$ powder of nominal purity $99.999 \%$. The powder was compacted under a compressive load to form disc-shaped samples of about $7 \mathrm{~mm}$ 


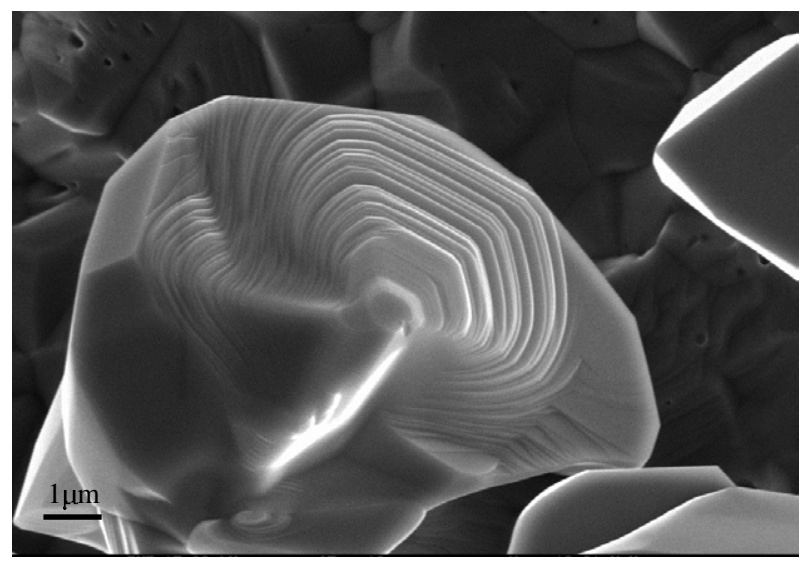

Figure 1. Protruding terrace structure appearing at the first stages of growth.

diameter and $2 \mathrm{~mm}$ thickness. The samples were then placed on an alumina boat near the gas inlet in a furnace, and sintered under argon flow at temperatures between 900 and $1300{ }^{\circ} \mathrm{C}$ for different times. It was found, however, that annealings in the range of $1250-1300{ }^{\circ} \mathrm{C}$ for $10 \mathrm{~h}$ lead to the growth of elongated structures on large areas of the discs, and therefore these conditions were applied. The argon flow was about $2 \mathrm{~m}^{-1}$, but this flow value is only relevant for the specific geometry of the furnace used. The morphology and size of the structures have been investigated by scanning electron microscopy in a Leica 440. Luminescence properties are investigated by means of cathodoluminescence in the SEM. The CL measurements were carried out at liquid nitrogen temperature on a Leica 440 or a Hitachi S-2500 SEM. CL images were recorded by using a Hamamatsu R928 photomultiplier while CL spectra were obtained with a Hamamatsu PMA-11 CCD camera.

\section{Results and discussion}

After treatments at temperatures below $1200^{\circ} \mathrm{C}$, some effects of sintering, such as increase of grain size and densification, were observed in the SEM, but no elongated structures appeared on the sample surface. At this temperature some grains with terraced morphology protrude from the surface (figure 1), which probably represents a preliminary stage of growth of the elongated structures. At temperatures between 1250 and $1300{ }^{\circ} \mathrm{C}$, elongated microstructures and nanostructures with different sizes and morphologies are formed.

The observed structure depends on the region of the sample and on the stage of formation. In the case of well developed structures, obtained after treatments at $1280^{\circ} \mathrm{C}$ or $1300^{\circ} \mathrm{C}$, the whole surface of the sample is covered by rods or wires as figure 2 shows. The influence of the argon flow is clearly observed in the figure. The gas inlet is at the right of the disc, causing the transport of evaporated material towards the left side, in which the longer structures, even with lengths in the millimetre range, are formed.

In previous stages of growth, the structures exhibit different morphologies, in addition to different sizes, across the sample. In the central part, sets of rods emerging in different directions from a common nucleation area are

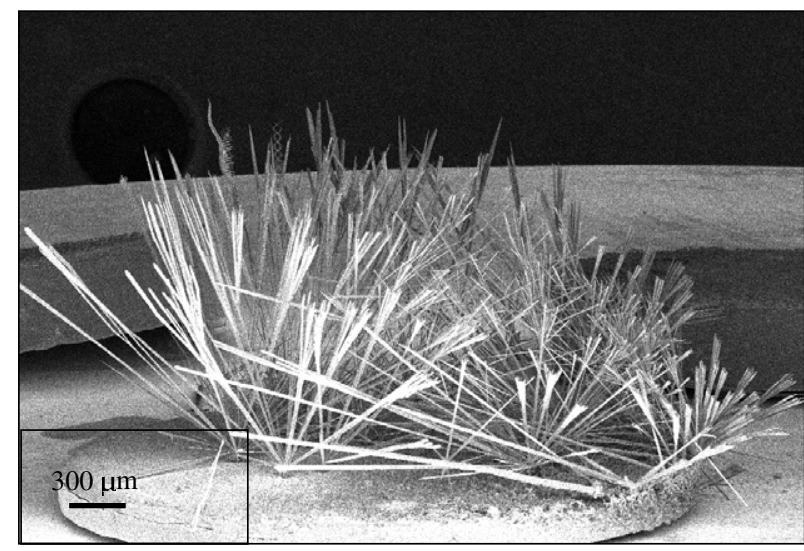

Figure 2. Sample covered with rods. The gas inlet is at the right of the image.

observed (figure 3(a)). SEM observation of different rods reveals different stages of growth as shown in figures 3(b) (d). Figure 3(b) shows part of a rod composed of a stacking sequence of crystallites perpendicular to the long axis, and figure 3(c) shows a higher magnification image of the rod in which some of the stacked crystallites appear connected by crystallites with pyramidal shape. Figure 3(d) shows a rod in which the facets and a pyramidal apex appear partially formed while in figure 3(e) a well formed hexagonal rod is observed. CL spectra recorded in the faces of a rod show a dominant emission at about $3.2 \mathrm{eV}$.

Besides these rods, with relatively large transverse dimensions, isolated needles as well as arrays of well oriented needles with diameter of about $100 \mathrm{~nm}$ are observed (figure 4) The root of these needles is formed by hexagonal prisms which become progressively thinner until they form bundles of needles in the top of the structure. Dense arrays of nanorods and needles with similar dimensions are formed in the rim of the disc shaped samples. In many cases, the needles join together forming the wall of a tubular structure as figure 5(a) shows. CL images reveal that the inner part of the tubes presents a higher luminescence than the outer wall (figure 5(b)). As stated in the introduction, thermal evaporation of powders under a gas flow, followed by deposition on a substrate, is an often reported method for obtaining elongated nanostructures of $\mathrm{ZnO}$. In many cases a catalyst, usually gold, is necessary, which leads to the formation of wires or belts with a gold tip, as a consequence of a vapour-liquid-solid (VLS) process $[18,19]$. In our case the source is a compacted disc which is also the substrate for the microstructures and nanostructures, which reduces the structure-substrate lattice mismatch problems. Since no catalytic process is involved it appears that the formation of the structures should take place by a vapour-solid process.

CL spectra of regions of the sample with and without needles are plotted in figure 6(a). Figure 6(b) shows CL spectra from the inner and outer surfaces of a tube, as well as the spectrum from untreated bulk $\mathrm{ZnO}$, which is represented for comparison. The spectra from the tube and the needles show an intense deep level band which corresponds to the well known green-orange band, and consists of several components in the range from 2 to $2.5 \mathrm{eV}$ associated with different point defects in the two sublattices and to impurities $([20,21]$ and references 
Growth and spatially resolved luminescence of low dimensional structures in sintered $\mathrm{ZnO}$
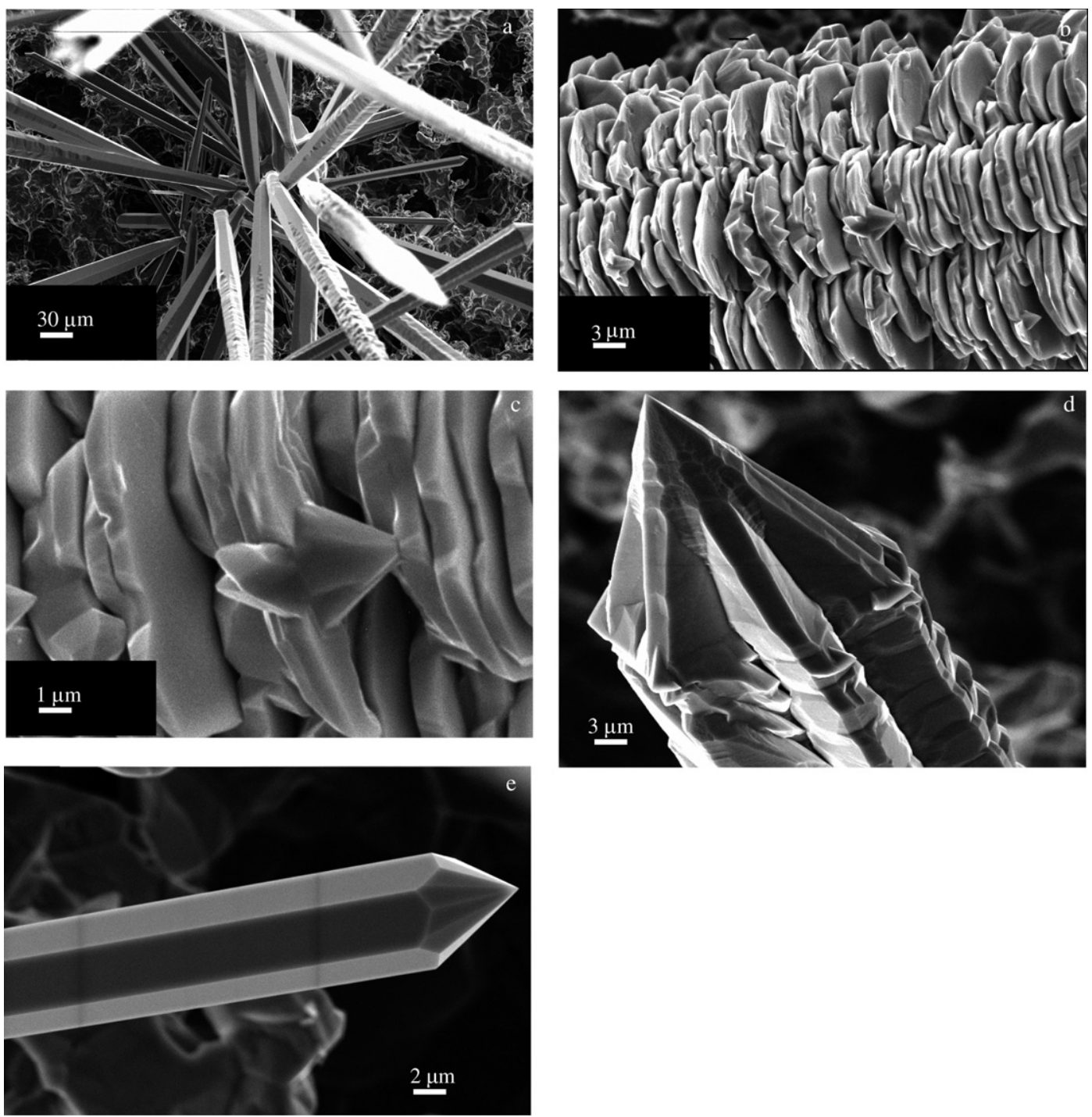

Figure 3. SEM images from the structures formed in the central part of the sample. (a)-(e) show rods at different stages of growth; (c) shows a detail of (b).

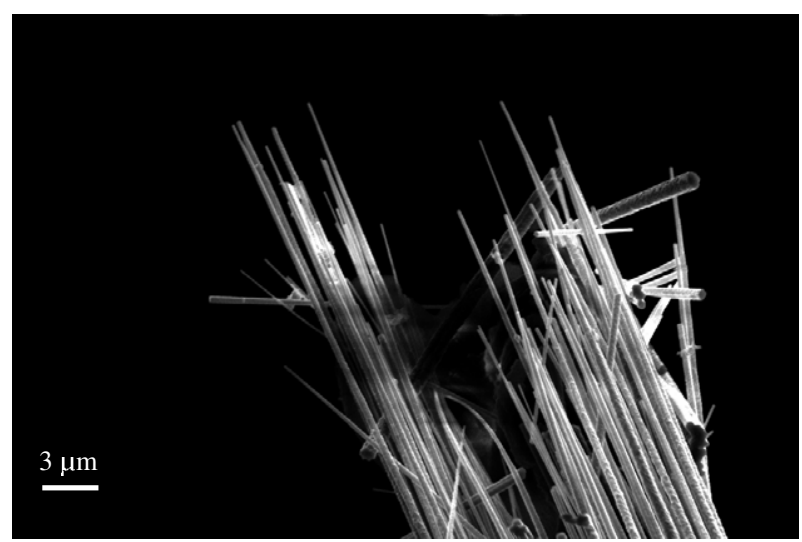

Figure 4. SEM image of a bundle of nanoneedles at the rim of the sample.

therein). The deep level band, in particular the green emission at about $2.38 \mathrm{eV}$, dominates the spectrum from the inner wall of the tube, which indicates the presence of a higher defect density in this surface. Though the origin of this emission is not definitely established, many authors relate it to centres involving oxygen vacancies $[22,23]$; thus a major role of this band could indicate a low oxygen content in the internal face of the tubes. Besides the increase of the deep level band, the presence of needles causes a reduction of the near band edge ultraviolet emission, which is often the case when new recombination paths are generated. However, the needles show a higher panchromatic CL emission than the background due to the broad intense green band associated with them.

In the part of the rim closest to the gas inlet, besides the above-described dense arrays of nanoneedles, structures with the forms of microcombs and feathers are grown. The combs (figure 7) consist of ribbons or plates, with a width of tens of microns and thickness of several microns, from which nanoneedles grow out in the lateral faces and preferentially on one side of the plate. A similar asymmetric growth of nanocantilevers in $\mathrm{ZnO}$ has been previously reported [24]. In that work, the role of the polarity in the stability of the surface 


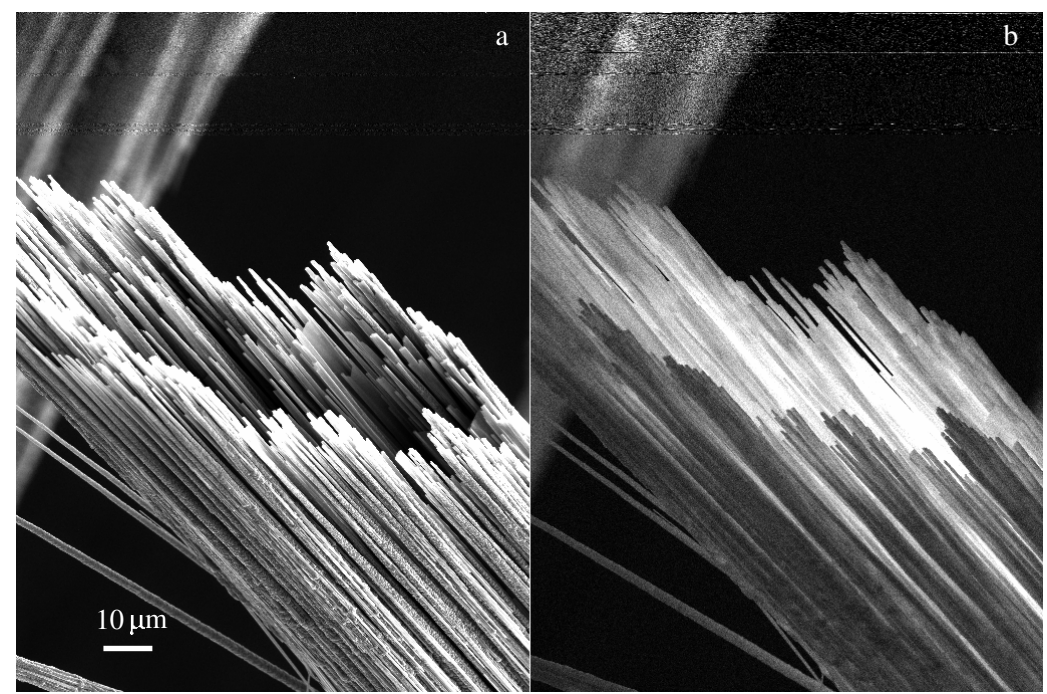

Figure 5. Tubular arrangement of nanoneedles: (a) secondary-electron and (b) CL images.
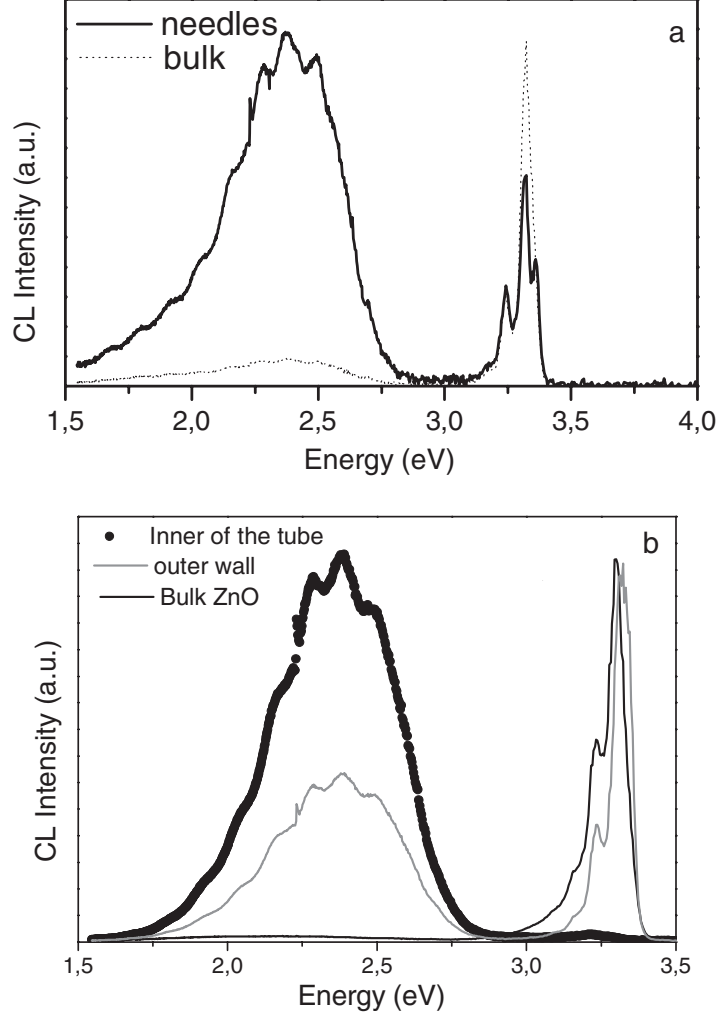

Figure 6. Comparison between the CL spectra obtained from: (a) bulk material and needle-like structures; (b) bulk material and tubular formations.

was established and it was found that the oxygen terminated [0001] surface would yield a lower growth ratio than the $\mathrm{Zn}$ terminated surface. Ab initio studies point also to the same conclusion. Wander et al [25] have estimated the surface energy of polar planes to be approximately twice that of the prismatic non-polar [1010]. According to these results, the asymmetric growth observed in figure 7 suggests that the lateral faces of the plate are polar planes and that the longer needles grow from the $\mathrm{Zn}$ terminated basal plane.
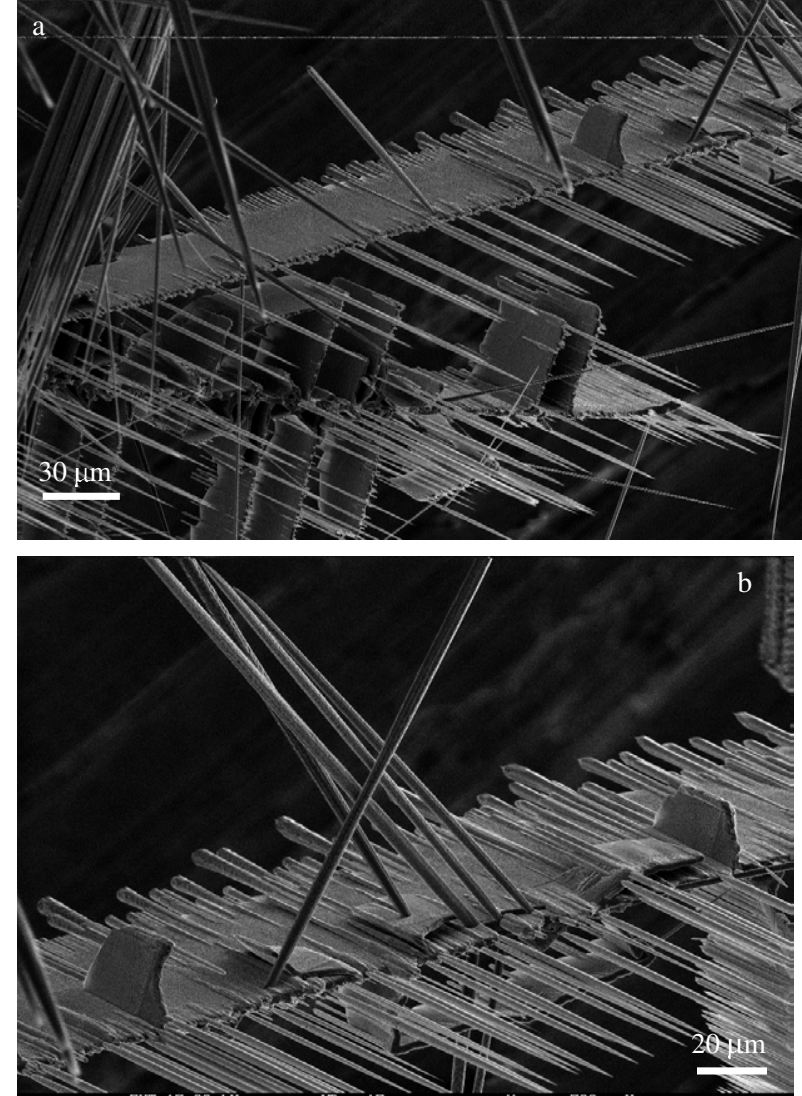

Figure 7. SEM images of: (a) comb-like structure; (b) a detail of the image shown in (a).

The structure of microfeathers is different from that of the combs. Instead of needles growing preferentially from one side of a ribbon, hexagonal nanotubes grow on both sides of a central axis (figure 8(a)). Many of these tubes show a complex distribution which includes a nanoneedle, emerging from the tubes, as shown in figure $8(\mathrm{~b})$.

In summary, sintering of compacted $\mathrm{ZnO}$ powder at temperatures between 1250 and $1300{ }^{\circ} \mathrm{C}$ under argon flow 
Growth and spatially resolved luminescence of low dimensional structures in sintered $\mathrm{ZnO}$
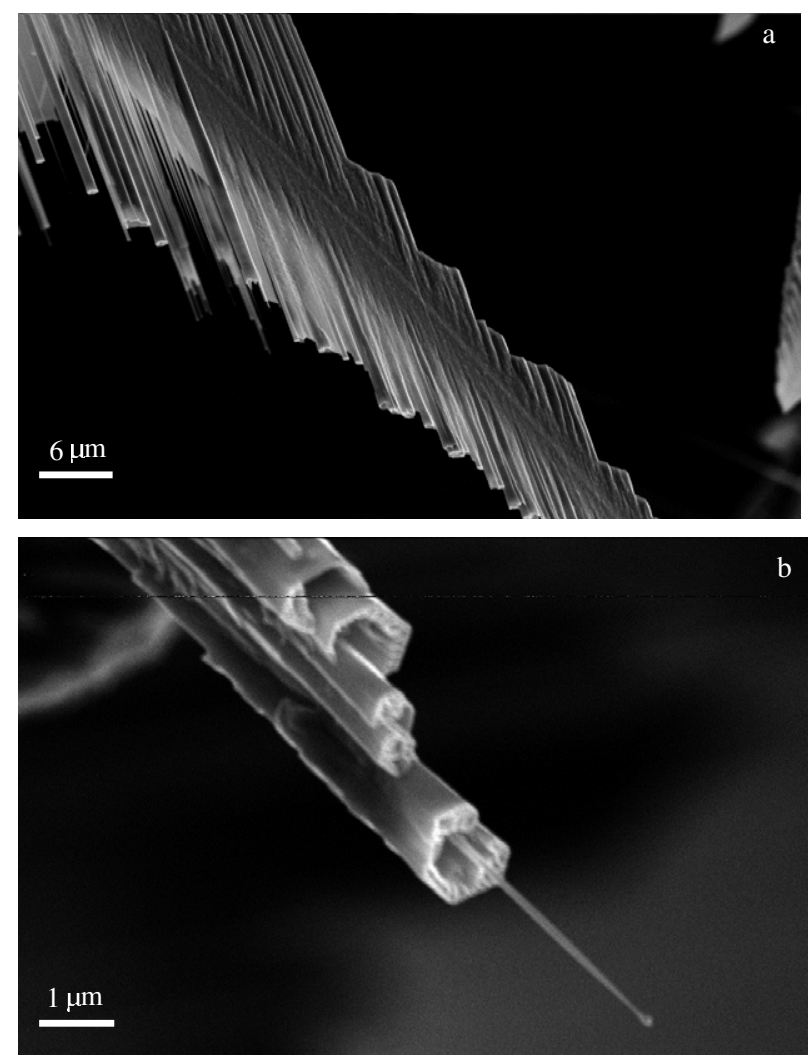

Figure 8. SEM images of: (a) feather-like structure; (b) detail of images shown in part (a).

leads to the growth of microstructures and nanostructures in the form of needles, tubes and rods on the sample surface. The elongated structures show a higher total CL emission than the sample background. In specific regions of the sample, microcombs and microfeathers containing well oriented arrays of nanoneedles are formed. CL spectra reveal different luminescence emission from the sample background as compared with that from the elongated structures. The structures show an intense emission of the deep level green band and a reduction of the near band edge emission. Enhanced CL emission has been observed in the inner wall of the tubes, as compared with the intensity emission of the outer surface.

\section{Acknowledgments}

This work was supported by MCYT (Project MAT200300455), by CAM (Project MAT/0630/2004) and by EU project
HPMT-CT-2001-00215. JG acknowledges the Marie Curie fellowship in the framework of this project (Grant HPMT-GH01-0021503).

\section{References}

[1] Pan Z W, Dai Z R and Wang Z L 2001 Science 2911947

[2] Huang M H, Mao S, Feick H, Yan H, Wu Y, Kind H, Weber E, Russo R and Yang P 2001 Science 2921897

[3] Hu J Q and Bando Y 2003 Appl. Phys. Lett. 821401

[4] Ng H T, Chen B, Li J, Han J, Meyyapan M, Wu J, Li S X and Haller E E 2003 Appl. Phys. Lett. 821689

[5] Zhao D, Liu Y, Shen D, Lu Y, Zhang L and Fan X 2003 J. Appl. Phys. 945605

[6] Baxter J B, Wu F and Aydil E S 2003 Appl. Phys. Lett. 833797

[7] Xing Y J, Xi Z H, Xue Z Q, Zhang X D, Song J H, Wang R M, Xu J, Song Y, Zhang S L and Yu D P 2003 Appl. Phys. Lett. 831689

[8] Lee J-S, Park K, Kang M-I, Park I-W, Kim S-W, Cho W K, Han H S and Kim S 2003 J. Cryst. Growth 254423

[9] Liu F, Gao P J, Zhang H R, Li J Q and Gao H J 2004 Nanotechnology 15949

[10] Zhang Y, Jia H and Yu D 2004 J. Phys. D: Appl. Phys. 37413

[11] Reynolds D C, Look D C and Jogai B 1996 Solid State Commun. 9973

[12] Reynolds D C, Look D C, Jogai B and Morkoç H 1997 Solid State Commun. 102643

[13] Zu P, Tang Z K, Wong G K L, Kawasaki M, Ohtomo A, Koinuma H and Segawa Y 1997 Solid State Commun. 103 459

[14] Zhao Q X, Willander M, Morjan R E, Hu Q H and Campbell E B 2003 Appl. Phys. Lett. 83165

[15] Dai Y, Zhang Y, Li Q K and Nan C W 2002 Chem. Phys. Lett. 35883

[16] Urbieta A, Fernández P and Piqueras J 2004 Appl. Phys. Lett. 855968

[17] Maestre D, Cremades A and Piqueras J 2005 J. Appl. Phys. 97 044316

[18] Wagner R S and Ellis W C 1964 Appl. Phys. Lett. 489

[19] Morales A M and Lieber C M 1998 Science 279208

[20] Radoi R, Fernández P, Piqueras J, Wiggins M S and Solis J 2003 Nanotechnology 14794

[21] Urbieta A, Fernández P, Piqueras J and Sekiguchi T 2001 Semicond. Sci. Technol. 16589

[22] Vanheusden K, Warren W L, Seager C H, Tallant D R, Voigt J A and Gnade B E 1996 J. Appl. Phys. 797983

[23] Roy V A L, Djurišić A B, Chan W K, Gao J, Lui H F and Surya C 2003 Appl. Phys. Lett. 83141

[24] Wang Z L, Kong X Y and Zuo J M 2003 Phys. Rev. Lett. 91 185502

[25] Wander A, Schedin F, Steadman P, Norris A, McGrath R, Turner T S, Thornton G and Harrison N M 2001 Phys. Rev. Lett. 863811 